\title{
Identification of quantitative trait loci for kernel traits in a wheat cultivar Chuannong16
}

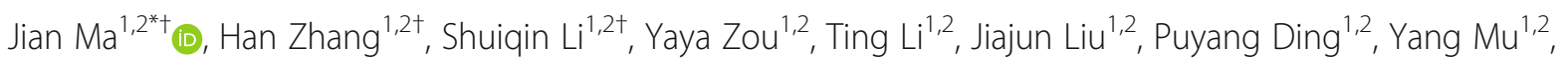
Huaping Tang ${ }^{1,2}$, Mei Deng ${ }^{1,2}$, Yaxi Liu ${ }^{1,2}$, Qiantao Jiang ${ }^{1,2}$, Guoyue Chen ${ }^{1,2}$, Houyang Kang ${ }^{1,2}$, Wei Li ${ }^{3}$, Zhien Pu ${ }^{3}$, Yuming Wei ${ }^{1,2}$, Youliang Zheng ${ }^{1,2}$ and Xiujin Lan ${ }^{1,2^{*}}$

\begin{abstract}
Background: Kernel length (KL), kernel width (KW) and thousand-kernel weight (TKW) are key agronomic traits in wheat breeding. Chuannong 16 ('CN16') is a commercial cultivar with significantly longer kernels than the line '20828'. To identify and characterize potential alleles from CN16 controlling KL, the previously developed recombinant inbred line (RIL) population derived from the cross '20828' $\times^{\prime} \mathrm{CN} 16^{\prime}$ and the genetic map constructed by the Wheat55K SNP array and SSR markers were used to perform quantitative trait locus/loci (QTL) analyses for kernel traits.

Results: A total of 11 putative QTL associated with kernel traits were identified and they were located on chromosomes 1A (2 QTL), 2B (2 QTL), 2D (3 QTL), 3D, 4A, 6A, and 7A, respectively. Among them, three major QTL, QKL.sicau-2D, QKW.sicau-2D and QTKW.sicau-2D, controlling KL, KW and TKW, respectively, were detected in three different environments. Respectively, they explained $10.88-18.85 \%, 17.21-21.49 \%$ and $10.01-23.20 \%$ of the phenotypic variance. Further, they were genetically mapped in the same interval on chromosome 2DS. A previously developed kompetitive allele-specific PCR (KASP) marker KASP-AX-94721936 was integrated in the genetic map and QTL re-mapping finally located the three major QTL in a 1- CM region flanked by AX-111096297 and KASP-AX94721936. Another two co-located QTL intervals for KL and TKW were also identified. A few predicted genes involved in regulation of kernel growth and development were identified in the intervals of these identified QTL. Significant relationships between kernel traits and spikelet number per spike and anthesis date were detected and discussed.

Conclusions: Three major and stably expressed QTL associated with KL, KW, and TKW were identified. A KASP marker tightly linked to these three major QTL was integrated. These findings provide information for subsequent fine mapping and cloning the three co-localized major QTL for kernel traits.
\end{abstract}

Keywords: Common wheat, Kernel traits, 55 K SNP array, QTL, KASP marker

\footnotetext{
* Correspondence: jianma@sicau.edu.cn; lanxiujin@163.com

†Jian Ma, Han Zhang and Shuiqin Li contributed equally to this work.

${ }^{1}$ Triticeae Research Institute, Sichuan Agricultural University, Chengdu

611130, Sichuan, China

Full list of author information is available at the end of the article
}

(c) The Author(s). 2019 Open Access This article is distributed under the terms of the Creative Commons Attribution 4.0 International License (http://creativecommons.org/licenses/by/4.0/), which permits unrestricted use, distribution, and reproduction in any medium, provided you give appropriate credit to the original author(s) and the source, provide a link to the Creative Commons license, and indicate if changes were made. The Creative Commons Public Domain Dedication waiver (http://creativecommons.org/publicdomain/zero/1.0/) applies to the data made available in this article, unless otherwise stated. 


\section{Background}

It is estimated that at least $2.4 \%$ of yield growth rate per year is required to meet food demand by 2050 due to the increasing world population [1]. However, crop yield increase rates have been so far unsatisfactory [2]. A better understanding and use of genetic determinants of kernel dimensions and weight could contribute to yield improvement in cereals [3]. Kernel weight is a major yield component principally defined by kernel length (KL), width (KW) and thickness [4]. Thus, it is valuable to identify and introduce favorable genes or alleles controlling kernel traits to improve yield in breeding.

Genes controlling kernel traits have been identified in tractable model species, such as Arabidopsis thaliana and Oryza sativa [5-7]. For example, qLGY3 encoding a MADS-domain transcription factor was associated with kernel size and could be modified to increase both kernel quality and yield potential in rice [8]. OsGW5 represents a major QTL controlling kernel width and weight in rice, and that it likely acts in the ubiquitin-proteasome pathway to control cell division during seed development [9]. The QTL qTGW3 encodes the GSK3/SHAGGY-like kinase OsGSK5/OsSK41 and interacts with OsARF4 to negatively regulate kernel size and weight in rice [7]. GS9 regulates kernel shape by altering cell division and improves the appearance quality of rice [10]. Using homology cloning, several orthologous genes associated with kernel traits have been isolated and characterized in common wheat (Triticum aestivum, AABBDD). For instance, TaGW2 [11] and TaGS5 [12] were isolated in wheat based on their orthologs with OsGW2 and OsGS5 in rice. TaGW2 was involved in regulation of KW, kernel weight, and kernel number in wheat [13]. TaGS5 was associated with thousand-kernel weight (TKW) [12], and TaGW8 was related to kernel size [14] in wheat.
In addition to isolating and characterizing wheat orthologs, numerous studies have focused on identifying quantitative trait locus/loci (QTL) for kernel traits. The detected QTL covered almost 21 chromosomes in wheat [15-20]. However, very few of them have been environmental-stably characterized and validated as the effects of QTL in hexaploid wheat are usually subtle in comparison with those identified in rice [21]. Large genetic distances between QTL and their flanking markers further restrict the utilization efficiency of kernel traits in wheat breeding.

In this study, we identified stably expressed QTL associated with KL, KW and TKW in a recombinant inbred line (RIL) mapping population developed from the cross between '20828' and Chuannong 16 ('CN16', 2CN) based on the constructed genetic map using the Wheat55K SNP array. A kompetitive allele-specific PCR (KASP) marker tightly linked to the major and stable QTL was developed and integrated in the genetic map and could be used in molecular breeding. The results reported here laid a foundation for subsequent fine mapping and cloning the three co-localized major QTL for kernel traits.

\section{Results}

Phenotypic evaluation and correlation analysis

The kernel traits of the $2 \mathrm{CN}$ population and their parents in different environments are listed in Table 1. 'CN16' had consistently and significantly higher values for KL than '20828', while '20828' is wider than 'CN16' in $\mathrm{KW}$ (Table 1, Fig. 1). For the $2 \mathrm{CN}$ population, the frequency distribution for kernel traits in all environments and best linear unbiased predictors (BLUP) showed continuous distributions with ranges from 5.33 to $8.07 \mathrm{~mm}$ in $\mathrm{KL}, 2.43$ to $4.19 \mathrm{~mm}$ in $\mathrm{KW}$ and 10.7 to $69.3 \mathrm{~g}$ in TKW (Table 1, Fig. 2). The broad-sense heritability of

Table 1 Phenotype of the parents and RILs in this study

\begin{tabular}{|c|c|c|c|c|c|c|c|c|c|}
\hline \multirow[b]{2}{*}{ Trait } & \multirow[b]{2}{*}{ Environment } & \multicolumn{2}{|l|}{ Parents } & \multicolumn{5}{|l|}{ RIL } & \multirow[b]{2}{*}{$h^{2}$} \\
\hline & & 20,828 & $\overline{C N 16}$ & $\overline{M i n}$ & Max & Mean & SD & $\mathrm{CV}$ & \\
\hline \multirow[t]{4}{*}{$\mathrm{KL}(\mathrm{mm})$} & $2017 C Z$ & $6.64^{b}$ & 7.16 & 5.97 & 8.07 & 7.06 & 0.42 & 0.06 & \multirow[t]{4}{*}{0.86} \\
\hline & 2017YA & $6.45^{b}$ & 6.84 & 5.33 & 7.81 & 6.53 & 0.44 & 0.07 & \\
\hline & 2018YA & $7.17^{\mathrm{b}}$ & 7.64 & 6.08 & 8.01 & 7.01 & 0.41 & 0.06 & \\
\hline & BLUP & 6.76 & 7.17 & 5.95 & 7.70 & 6.86 & 0.34 & 0.05 & \\
\hline \multirow[t]{4}{*}{$\mathrm{KW}(\mathrm{mm})$} & $2017 C Z$ & $3.84^{b}$ & 3.67 & 3.24 & 4.17 & 3.80 & 0.18 & 0.05 & \multirow[t]{4}{*}{0.64} \\
\hline & 2017YA & $3.54^{b}$ & 3.51 & 2.64 & 3.94 & 3.46 & 0.20 & 0.06 & \\
\hline & 2018YA & $4.01^{a}$ & 3.85 & 2.43 & 4.19 & 3.63 & 0.32 & 0.09 & \\
\hline & BLUP & 3.74 & 3.66 & 3.29 & 3.92 & 3.63 & 0.12 & 0.03 & \\
\hline \multirow[t]{4}{*}{ TKW (g) } & $2017 C Z$ & 43.5 & 44.7 & 16 & 69.3 & 44.5 & 0.75 & 0.17 & \multirow[t]{4}{*}{0.73} \\
\hline & 2017YA & $53.7^{b}$ & 51.7 & 31 & 67 & 51.5 & 0.58 & 0.11 & \\
\hline & 2018YA & 43.2 & 44.2 & 10.7 & 66.8 & 41.9 & 0.67 & 0.16 & \\
\hline & BLUP & 46.6 & 46.6 & 33.7 & 60.8 & 46 & 0.43 & 0.09 & \\
\hline
\end{tabular}

${ }^{a}$ Difference is significant at the 0.05 level, ${ }^{b}$ Difference is significant at the 0.01 level 

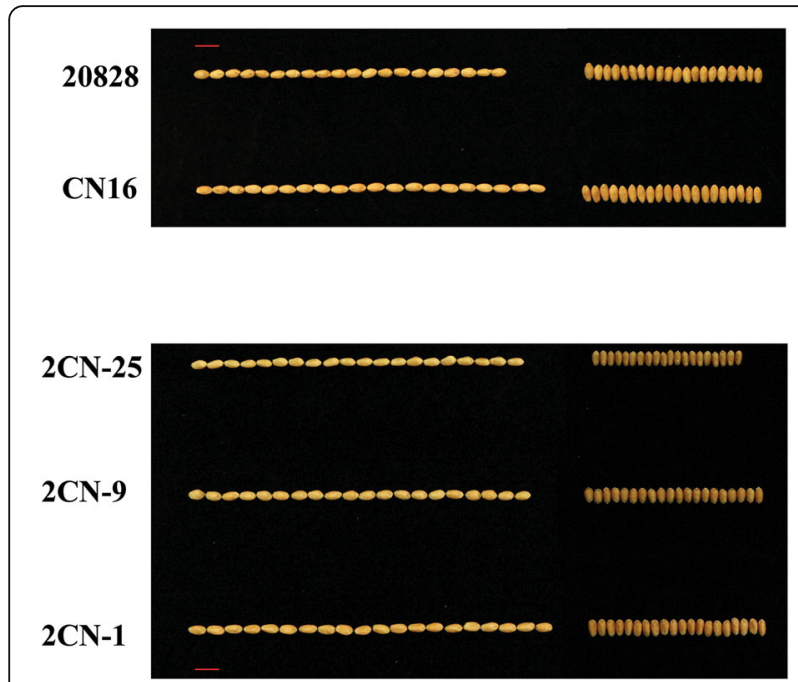

Fig. 1 Kernel phenotypes of the parent '20828', 'CN16' and partial RILs. The red line represents the scale $=1 \mathrm{~cm}$

KL, KW and TKW were $0.86,0.64$ and 0.73 , respectively (Table 1).

Correlation analysis showed that KL, KW and TKW among different environments were all significant, and the correlation coefficients ranged from 0.38 to 0.92 $(P<0.01$, Additional file 2: Table S1). Significant correlations with coefficients ranging from 0.48 to 0.83 among all three kernel traits based on the BLUP data were detected as well $(P<0.01$, Additional file 3: Table S2).

Moreover, the phenotypic correlation analyses between the investigated kernel traits and other agronomic traits showed that all three kernel traits had significantly and negatively correlations with spikelet number per spike and anthesis date $(P<0.01)$. KW was significantly and positively correlated with plant height $(P<0.01)$ and significant correlations were also detected between TKW and plant height $(P<0.05$, Additional file 4 : Table S3).

\section{QTL mapping}

A total of 11 putative QTL associated with kernel traits were detected in the $2 \mathrm{CN}$ population (Table 2). They were located on chromosomes 1A (2 QTL), 2B (2 QTL), 2D (3 QTL), 3D, 4A, 6A, and 7A (Table 2, Fig. 3). Of them, six QTL conferring KL were identified individually explaining $2.57-18.05 \%$ of the phenotypic variance. QKL.sicau-2D, as a major locus, was detected in all environments and explained $10.88-18.05 \%$ of the phenotypic variance. The positive allele at QKL.sicau- $2 D$ was derived from 'CN16' (Table 2). QKL.sicau-6A was detected in two environments. This locus explained 5.16-7.09\% of phenotypic variance. The positive allele at this QTL was derived from '20828'.

Two QTL conferring KW, QKW.sicau-2D and QKW.sicau-3D were detected and accounted for $5.15-21.49 \%$ of
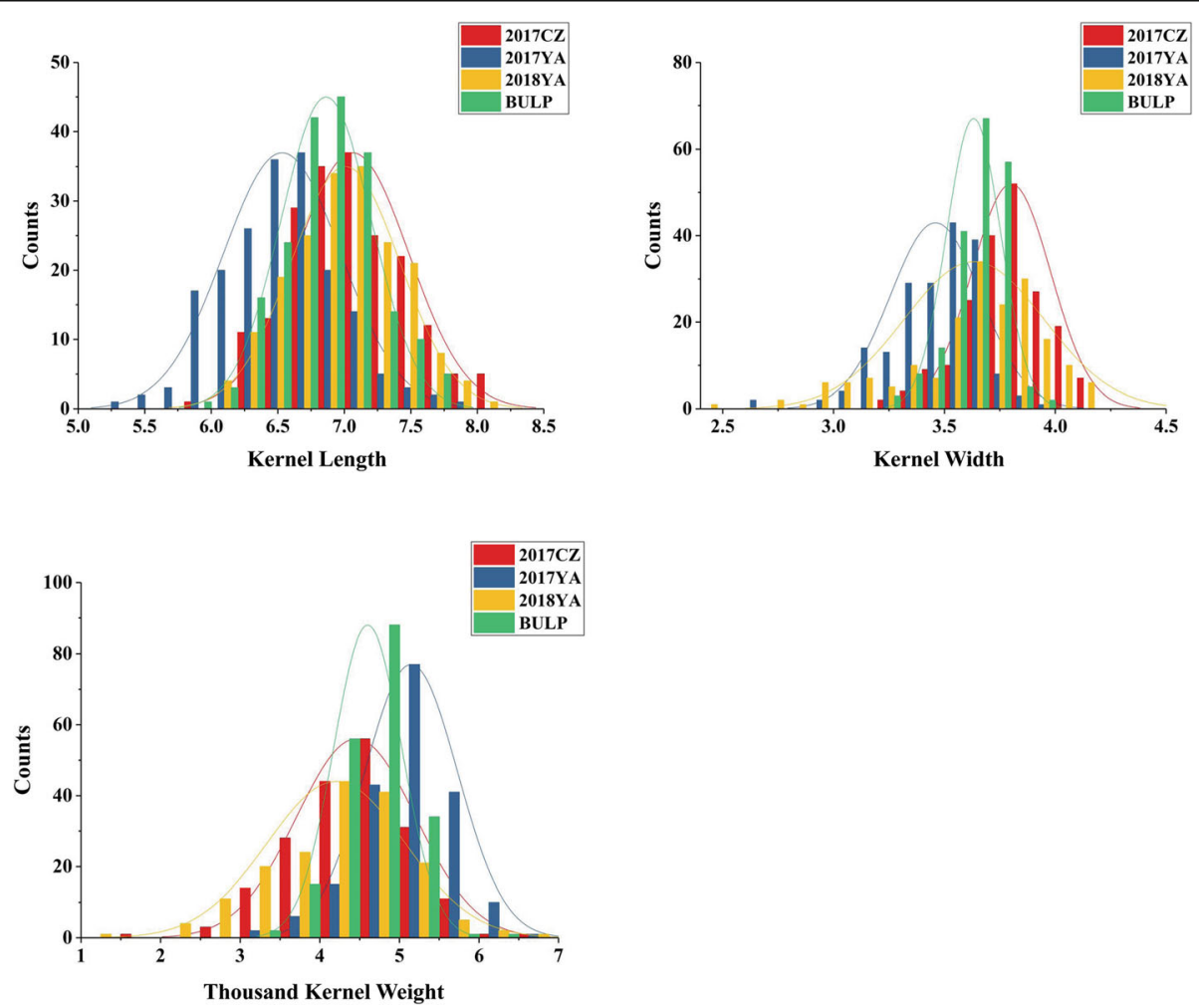

Fig. 2 Frequency distribution of three kernel traits in $2 \mathrm{CN}$ population in different environments 
Table 2 Quantitative trait loci for kernel traits identified in the '20828' × 'CN16' population evaluated in different environments

\begin{tabular}{|c|c|c|c|c|c|c|c|c|c|}
\hline Trait & QTL & Environment & Chromosome & Interval (cM) & Left Marker & Right Marker & LOD & PVE (\%) & Add \\
\hline \multirow[t]{16}{*}{$\overline{\mathrm{KL}}$} & \multirow[t]{3}{*}{ QKL.sicau-1A } & $2017 C Z$ & \multirow[t]{3}{*}{$1 \mathrm{~A}$} & $167.5-172.5$ & $A X-111476496$ & $A X-110466836$ & 7.12 & 5.21 & -0.10 \\
\hline & & 2017YA & & $167.5-171.5$ & $A X-109558656$ & $A X-111476496$ & 4.98 & 6.59 & -0.11 \\
\hline & & BLUP & & $167.5-171.5$ & $A X-109558656$ & $A X-111476496$ & 5.28 & 4.00 & -0.08 \\
\hline & \multirow[t]{2}{*}{ QKL.sicau-2B } & 2018YA & \multirow[t]{2}{*}{$2 B$} & $63.5-64.5$ & $A X-110425276$ & $A X-111616168$ & 6.11 & 7.99 & 0.13 \\
\hline & & BLUP & & $63.5-64.5$ & $A X-110425276$ & $A X-111616168$ & 6.98 & 5.24 & 0.09 \\
\hline & \multirow[t]{4}{*}{ QKL.sicau-2D } & $2017 C Z$ & \multirow[t]{4}{*}{$2 \mathrm{D}$} & $67.5-68.5$ & $A X-111096297$ & KASP-AX-94721936 & 21.13 & 18.05 & -0.20 \\
\hline & & 2017YA & & $67.5-68.5$ & AX-1111096297 & KASP-AX-94721936 & 11.91 & 17.11 & -0.18 \\
\hline & & 2018YA & & $67.5-68.5$ & $A X-111096297$ & KASP-AX-94721936 & 8.17 & 10.88 & -0.15 \\
\hline & & BLUP & & $67.5-68.5$ & $A X-111096297$ & KASP-AX-94721936 & 17.62 & 14.95 & -0.15 \\
\hline & \multirow[t]{2}{*}{ QKL.sicau-4A } & 2018YA & \multirow[t]{2}{*}{$4 \mathrm{~A}$} & $157.5-158.5$ & $A X-110030140$ & $A X-110132746$ & 7.70 & 10.00 & -0.15 \\
\hline & & BLUP & & $157.5-158.5$ & $A X-110030140$ & $A X-110132746$ & 7.01 & 5.27 & -0.09 \\
\hline & \multirow[t]{3}{*}{ QKL.sicau-6A } & $2017 C Z$ & \multirow[t]{3}{*}{$6 \mathrm{~A}$} & $6.5-7.5$ & $A X-109498898$ & $A X-111594929$ & 6.91 & 5.16 & 0.10 \\
\hline & & 2017YA & & $7.5-11.5$ & AX-109498898 & $A X-111594929$ & 5.24 & 7.09 & 0.11 \\
\hline & & BLUP & & $7.5-10.5$ & $A X-109498898$ & $A X-111594929$ & 3.50 & 2.57 & 0.06 \\
\hline & \multirow[t]{2}{*}{ QKL.sicau-7A } & 2017YA & \multirow[t]{2}{*}{$7 \mathrm{~A}$} & $0-2.5$ & $A X-109868790$ & $A X-110460191$ & 5.92 & 7.81 & -0.12 \\
\hline & & BLUP & & $0-1.5$ & $A X-109868790$ & $A X-110460191$ & 7.26 & 5.40 & -0.09 \\
\hline \multirow[t]{5}{*}{ KW } & \multirow[t]{3}{*}{ QKW.sicau-2D } & $2017 C Z$ & \multirow[t]{3}{*}{$2 \mathrm{D}$} & $67.5-68.5$ & $A X-111096297$ & KASP-AX-94721936 & 10.35 & 17.21 & -0.07 \\
\hline & & 2018YA & & $63.5-67.5$ & $A X-111096297$ & KASP-AX-94721936 & 10.24 & 17.35 & -0.14 \\
\hline & & BLUP & & $67.5-68.5$ & AX-1111096297 & KASP-AX-94721936 & 14.46 & 21.49 & -0.06 \\
\hline & \multirow[t]{2}{*}{ QKW.sicau-3D } & 2018YA & \multirow[t]{2}{*}{$3 \mathrm{D}$} & $56.5-70.5$ & $A X-1111126228$ & $A X-110987465$ & 3.29 & 5.15 & 0.08 \\
\hline & & BLUP & & $56.5-71.5$ & $A X-110987465$ & $A X-110543919$ & 5.72 & 8.49 & 0.03 \\
\hline \multirow[t]{8}{*}{ TKW } & \multirow[t]{2}{*}{ QTKW.sicau-1A } & $2017 C Z$ & \multirow[t]{2}{*}{$1 \mathrm{~A}$} & $167.5-171.5$ & $A X-109558656$ & $A X-111476496$ & 4.59 & 7.13 & -0.20 \\
\hline & & BLUP & & $167.5-171.5$ & $A X-111476496$ & $A X-110466836$ & 3.24 & 4.01 & -0.09 \\
\hline & \multirow[t]{2}{*}{ QTKW.sicau-2B } & $2017 C Z$ & \multirow[t]{2}{*}{$2 \mathrm{~B}$} & $63.5-64.5$ & $A X-110425276$ & $A X-111616168$ & 5.30 & 8.25 & 0.22 \\
\hline & & BLUP & & $63.5-64.5$ & $A X-110425276$ & $A X-111616168$ & 3.81 & 4.80 & 0.10 \\
\hline & \multirow[t]{4}{*}{ QTKW.sicau-2D } & $2017 C Z$ & \multirow[t]{4}{*}{$2 \mathrm{D}$} & $67.5-68.5$ & $A X-111096297$ & KASP-AX-94721936 & 7.38 & 11.57 & -0.25 \\
\hline & & 2017YA & & $67.5-68.5$ & $A X-111096297$ & KASP-AX-94721936 & 9.57 & 13.30 & -0.23 \\
\hline & & 2018YA & & $67.5-68.5$ & $A X-111096297$ & KASP-AX-94721936 & 6.77 & 10.01 & -0.32 \\
\hline & & BLUP & & $67.5-68.5$ & $A X-111096297$ & KASP-AX-94721936 & 16.38 & 23.2 & -0.2 \\
\hline
\end{tabular}

the phenotypic variance. $Q K W$.sicau- $2 D$ was a major locus and explained $17.21-21.49 \%$ of the phenotypic variance in three environments and the BLUP dataset. The positive allele at it was derived from 'CN16' (Table 2).

Three QTL associated with TKW were detected with $4.01-23.20 \%$ of the phenotypic variance explained. A major QTL, QTKW.sicau-2D, accounting for 10.01$23.20 \%$ of the phenotypic variance, was stably detected in three environments and the BLUP dataset. The positive allele at this QTL was contributed by 'CN16' (Table 2).

The remaining 8 QTL including QKL.sicau-1A, QKL.sicau-2B, QKL.sicau-4A, QKL.sicau-6A, QKL.sicau-7A, QTKW.sicau-1A, QTKW.sicau-2B and QKW.sicau-3D explained less than $10 \%$ of the phenotypic variance and could only be detected in less than two environments (Table 2).

Interestingly, the three major and stable QTL, QKL.sicau$2 D$ for KL, QKW.sicau-2D for KW, and QTKW.sicau- $2 D$ for TKW, were co-located in the same interval between 67.5 and $68.5 \mathrm{cM}$. QKL.sicau-1A for KL and QTKW.sicau$1 A$ for TKW were co-located in the interval between 167.5 and $172.5 \mathrm{cM}$. QKL.sicau- $2 B$ for KL and QTKW.sicau- $2 B$ for TKW were also co-located in the interval between 63.5 and $64.5 \mathrm{cM}$ (Table 2, Fig. 3). These three co-located QTL intervals suggest that there may be a major QTL with pleiotropic effects affecting related traits or a cluster of linked QTL that affect multiple various traits [22, 23].

QTL $\times$ environment $(\mathrm{QE})$ interaction analysis showed that a total of 45 QTL were detected (Additional file 5: Table S4). Eleven of these QTL were the same as those detected in individual environment QTL mapping. For instance, QKL.sicau-2D, QKW.sicau-2D and QTKW.sicau-2D were all detected, further indicating that they were major and stable. The remaining QTL showed low LOD scores and low 


$\begin{array}{lllllll}\text { chr1A } & \text { chr2B } & \text { chr2D } & \text { chr3D } & \text { chr4A } & \text { chr6A } & \text { chr7A }\end{array}$
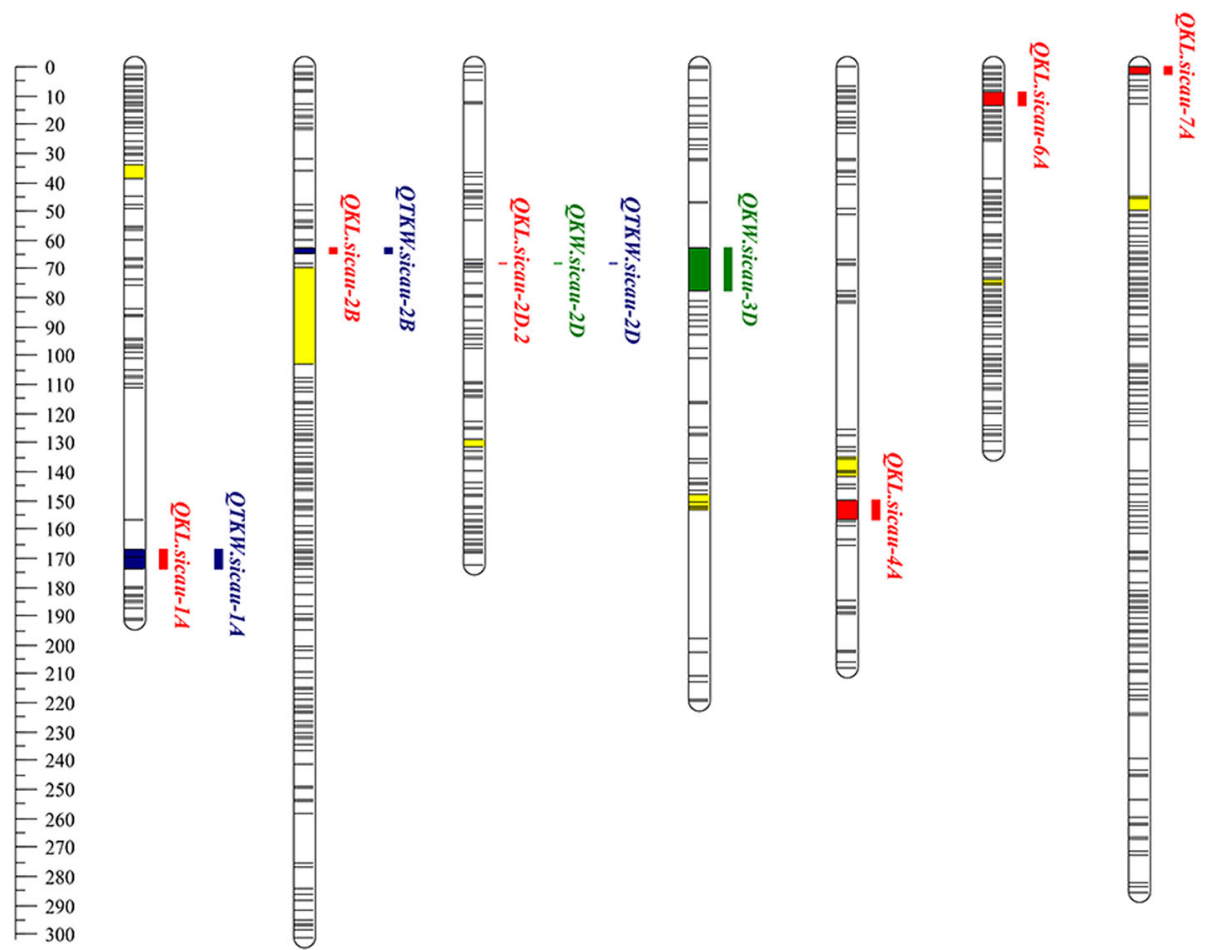

Fig. 3 Eleven putative and stable QTL for kernel traits in the genetic map. Red color represents QTL conferring KL, green color represents QTL conferring KW, blue color represents QTL conferring TKW, and the centromere was indicated in yellow color

phenotypic variance explained in the QE interaction analysis.

\section{Marker development and validation}

The co-located interval for the three major QTL, QKL.sicau-2D, QKW.sicau-2D and QTKW.sicau-2D were firstly mapped between $A X-111096297$ and $A X-86171316$ (Fig. 4). SNPs in this region detected by the Wheat660K array in the parents of the $2 \mathrm{CN}$ population were converted to KASP markers [24]. KASP-AX-94721936 was genetically mapped between $A X-111096297$ and $A X-86171316$ (Fig. 4). Finally, QTL re-mapping showed that QKL.sicau-2D, QKW.sicau$2 D$ and $Q T K W$.sicau- $2 D$ were all located between $A X$ 111096297 and KASP-AX-94721936 (Fig. 4). In addition, $A X-111096297$ and KASP-AX-94721936 were used to BLAST against the pseudomolecules of 'Chinese Spring' ('CS'). BLAST results showed that $A X-111096297$ and KASP-AX-94721936 were located at $32.97 \mathrm{Mbp}$ and $33.74 \mathrm{Mbp}$, respectively, in the deletion bin of 2DS5-0.471.00 on the short arm of chromosome 2DS (Fig. 4).

The homozygous lines of parental alleles '20828' and 'CN16' at each QKL.sicau-2D, QKW.sicau-2D and QTKW.sicau-2D were selected based on the genotyping data of $A X-111096297$ and KASP-AX-94721936 for the $2 \mathrm{CN}$ population. $T$-test showed that the lines carrying the ' $\mathrm{CN} 16$ ' alleles had significantly higher phenotypic values than those carrying the '20828' alleles at all the three QTL in different environments and the BLUP datasets $(P<0.05$, Fig. 5).

\section{Detection of the effect of 1BL/1RS translocation on kernel traits}

No significant difference was detected between lines carrying $1 \mathrm{BS}$ and $1 \mathrm{RS}$ for KL, KW and TKW (Additional file 1: Figure S1). This result likely indicated that there is no QTL affecting these kernel traits on the 1BS or 1RS chromosome arm.

\section{Discussion}

Aegilops tauschii, as the donor of the D subgenome, provides an important genetic resource for hexaploid wheat [25-27]. Zhao et al. [28] integrated a large amount of key agronomic genes/QTL and anchored them to the physical map of Ae. tauschii. Their results suggested that the $\mathrm{D}$ genome, especially for chromosomes 2D and 7D, has made strong positive contributions to wheat improvement. Thus, studies on the D genome are meaningful for 


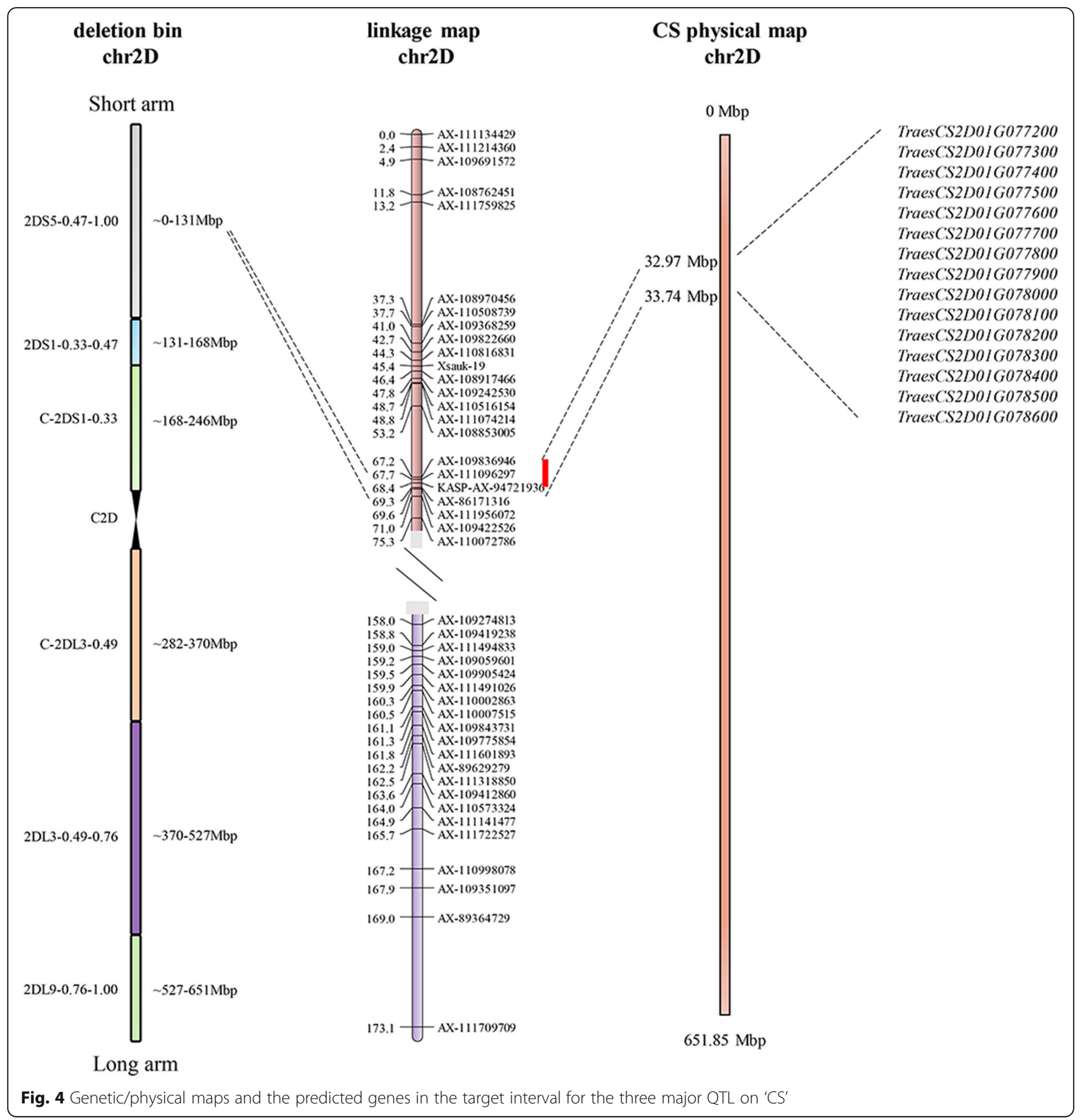

understanding evolution and domestication of wheat. $\mathrm{Nu}$ merous studies have revealed a large number of QTL for kernel traits on 2D [16, 19, 20, 29-36]. Here, three major stable QTL, QKL.sicau-2D, QKW.sicau-2D and QTKW.sicau-2D, associated with KL, KW and TKW, respectively, were detected on the short arm of chromosome 2DS. These results further indicated that chromosome 2D likely contributed positively to kernel traits and yield.

Comparison of physical intervals showed that the major QTL QKL.sicau-2D and QKW.sicau-2D were mapped in different intervals from those detected previously on chromosome 2D (Additional file 6: Table S5). However, we found that QTKW.sicau-2D was co-located with the locus for TKW reported by $\mathrm{Yu}$ et al. [19], suggesting they are likely alleles. Given the co-located of the three major QTL for KL (QKL.sicau-2D), KW (QKW.sicau-2D), and TKW (QTKW.sicau-2D) in this study, QTKW.sicau-2D may have a different role from that reported in Yu et al. [19]. These three major QTL were mapped in the same region between 32.97Mbp and 33.74Mbp covering 0.77 Mbp. There were 15 genes in this interval, and 11 of them were likely associated with kernel traits (Additional file 7: Table S6, Fig. 4). 

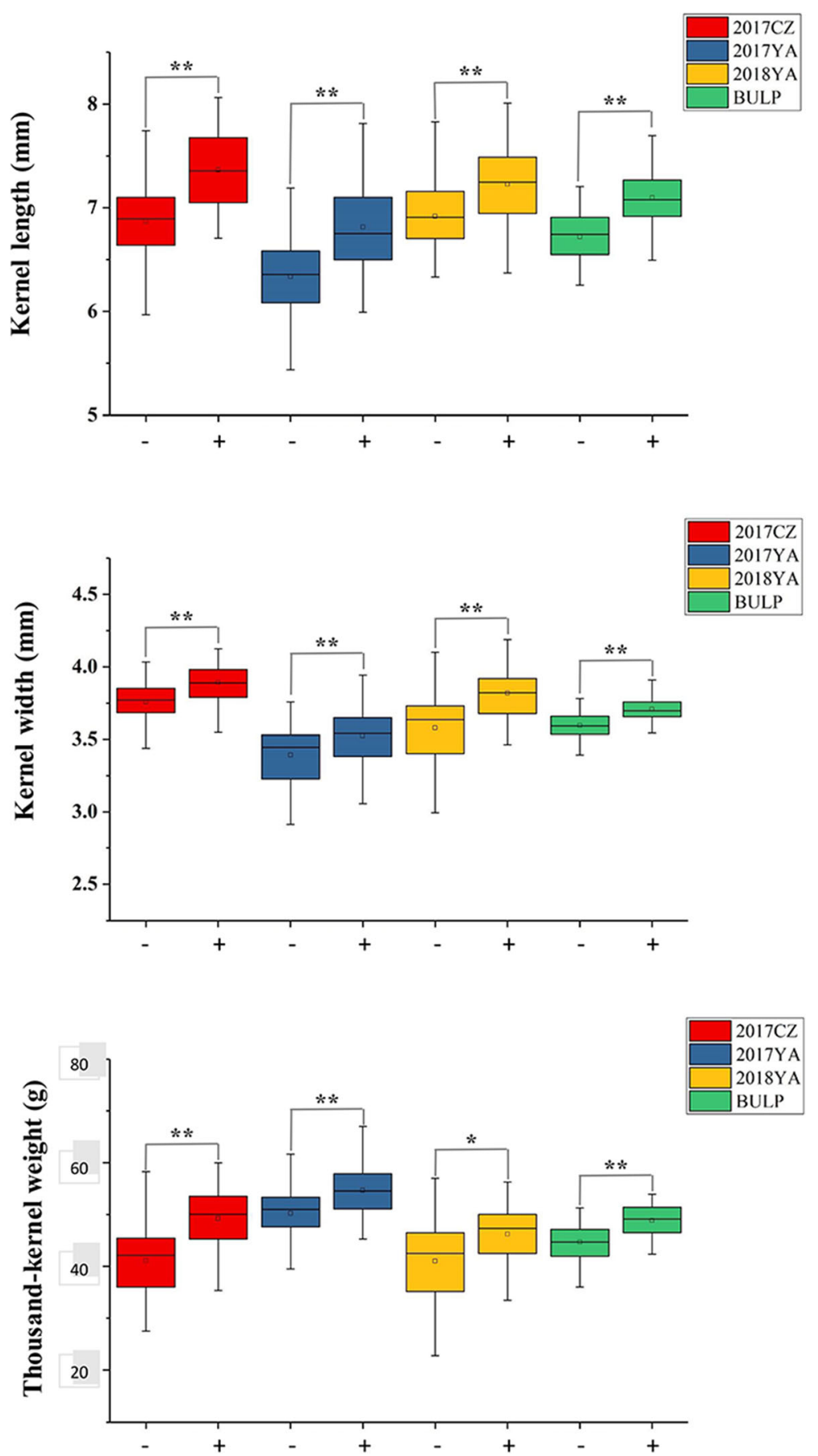

Fig. 5 Effects of QKL.sicau-2D, QKW.sicau-2D and QTKW.sicau-2D in 2CN population. '-' represents the homozygous lines of '20828' allele, ' + ' represents the homozygous lines of 'CN16' allele, * Significance at the 0.05 probability level, **Significance at the 0.01 probability level

For example, TraesCS2D01G077400 encodes an actin crosslinking protein and was detected to be mainly expressed in pollen of Arabidopsis [37], thus likely affecting kernel development. TraesCS2D01G077900 encodes a DnaJ domain containing protein and reacts on the polar nuclear fusion further affecting endosperm proliferation in Arabidopsis thaliana [38].
In addition to the major QTL, we also identified a few minor QTL expressed in a single or two environment(s). These minor and unstable QTL could be mostly affected by environmental factors and may not be always expressed. The co-located interval (581.42 Mbp-584.83 Mbp on 1AL) for QKL.sicau-1A and QTKW.sicau-1A was overlapped with the co-located cluster for QKl.ncl-1A.1 
and QTkw.ncl-1A.1 [39], suggesting they may be allelic (Additional file 6: Table S5). There were 73 predicted genes in this interval (Additional file 7: Table S6). The colocated interval (67.47 Mbp-72.59 Mbp on 2BS) for QKL.sicau-2B and QTKW.sicau-2B was overlapped with QTkw-2B.3 [20] and QTgw.crc-2B [29] for TKW, indicating they may be alleles. There were 43 predicted genes in this interval (Additional file 7: Table S6). QKL.sicau-7A was overlapped with QGl.cau-7A.1 [11] and the locus flanked by $w P t-0321$ and Xbarc121 [40], and thus they were likely alleles (Additional file 6: Table S5). There were 21 predicted genes in this interval (8.24 Mbp - 8.39 Mbp on 7AS). QKL.sicau-4A and QKL.sicau-6A were not overlapped with previously identified QTL for KL, suggesting they might be new loci (Additional file 6: Table S5). There were 131 and 71 predicted genes, respectively, in their located intervals (41.74 Mbp - 60.36 Mbp on 4AL and 6.27 Mbp-9.48 Mbp on 6AS). Few QTL for KW have been reported, and comparison of QKW.sicau-3D with those identified in previous studies showed no overlapped intervals. There were 80 predicted genes in this interval (573.10 Mbp - 578.25 Mbp on 3DL). For the predicted genes in the intervals of these minor and unstable QTL, a few were involved in growth and development of kernel. For instance, TraesCS7A01G129200 encodes an F-box family protein. F-box protein is known to be involved in the nutrient and reproductive growth and development of many plants, and can function as a site of protein-protein interaction providing a basis for grain grouting [41]. TraesCS3D01G474800 encodes an expansin protein. Previous studies showed that expansin proteins are cell wall proteins [42], and they can regulate plant growth through controlling cell extension via the disruption of hydrogen bonds between matrix glucans and cellulose. TraesCS6A01G015200 encodes a mitochondrial transcription termination factor, which can promote embryo and endosperm development, resulting in large kernels [43].

In the present study, although the KW value of ' $\mathrm{CN} 16$ ' was lower than that of '20828', we detected one major QTL QKW.sicau-2D at which the positive allele was contributed from 'CN16' and only a minor QTL QKW.sicau-3D with lower explained phenotypic variance at which the positive allele was contributed from '20828'. Similar findings are not uncommon. In previous QTL analysis, positive effects at QTL have frequently been contributed by the lower-value parents. For example, although the phenotypes of KW and TKW in parents of YN15 and SJZ54 were lower than those of M8008, the effects of the identified QTL for KW and TKW were increased by YN15 and SJZ54 alleles [44]. Breseghello and Sorrells [45] identified a major QTL on chromosome 2D for grain weight linked with SSR marker wmc18. Despite the parent AC Reed showed larger seeds than the other parent Grandin, the Grandin allele at wmc18 was responsible for an increase of approximately $1.5 \mathrm{mg}$ kernel $^{-1}$ [45]. The parent '20828' likely possesses more than one allele that contributes to the formation of wider kernel. As the low coverage of SNP array around the centromere of chromosomes may lead to the lack of mapped markers on these genetic regions [46]. Thus, we cannot rule out the possibility that other QTL for KW at which the positive alleles are from '20828' might be located around the centromere where the genetic map was absent in this study.

In this study, positive and significant correlations among all the three kernel traits were detected (Additional file 3: Table S2). Similar results were reported in previous studies $[35,39,45]$. This suggests that selection for larger kernels was accompanied by selection for heavier kernels during domestication and breeding process [39]. KL, KW and TKW were all significantly and negatively correlated with spikelet number per spike and anthesis date (Additional file 4: Table S3). QTL mapping indicated that major QTL for spikelet number per spike [24] and anthesis date were co-located with the major QTL for KL, KW and TKW (Additional file 8: Table S7), further confirming their close relationships. It is well known that for a single spike, an increase in spikelet number is usually accompanied with reduced kernel weight due to nutrition competition [47-49]. This was also clearly manifested by the reciprocal action of the parental alleles at a co-located interval for spikelet number per spike and TKW (Additional file 8: Table S7). The alleles from '20828' increased spikelet number per spike, while the corresponding alleles from 'CN16' increased TKW. TKW and KW were significantly and positively correlated with plant height (Additional file 4: Table S3). The physical positions of QKW.sicau-2D and $Q T K W$.sicau-2D were far away from the dwarfing gene $R h t 8$ [50] for plant height on the physical map of 'CS'. Thus, there may be a potentially other pleiotropic locus controlling TKW and plant height as indicated by QTL mapping (Additional file 8: Table S7).

Functional markers have been effectively applied in some breeding programs [51, 52]. Molecular markers should possess the feature of high-throughput and costeffectiveness [53]. With the decrease of sequencing cost, a large amount of SNPs have been identified. Given its advantages, KASP marker has been widely applied in wheat genetics and breeding. Here, the developed KASP marker will be helpful for further selection of heterozygous lines for developing near-isogenic lines and QTL validation in different backgrounds.

\section{Conclusion}

In this paper, we identified three major and stably expressed and eight minor QTL associated with kernel traits based on the linkage map constructed by the Wheat55K SNP array. Three co-located intervals for 
kennel traits were identified. One was located on the short arm of chromosome 2D containing the three major and likely novel QTL conferring KL, KW and TKW, respectively. The other two both containing minor QTL for KL and TKW were located on chromosomes $1 \mathrm{~A}$ and $2 \mathrm{~B}$, respectively. A few genes involved in regulation of kernel growth and development were identified in the intervals of these identified QTL. A KASP marker tightly linked the three major QTL would be useful for subsequent fine mapping and molecular marker selection breeding.

\section{Methods}

\section{Plant materials and field environments}

A RIL mapping population containing $199 \mathrm{~F}_{6}$ lines was developed from the cross between '20828' and 'CN16'. 'CN16' is a commercial cultivar with strong tillering and suitable plant type. The line '20828', with high level of resistance to rust, has been widely utilized as a crossing parent in wheat breeding.

The $2 \mathrm{CN}$ population was planted at Chongzhou $\left(103^{\circ}\right.$ $\left.38^{\prime} \mathrm{E}, 30^{\circ} 32^{\prime} \mathrm{N}\right)$ in 2017 (2017CZ) and Ya'an (103'0 $0^{\prime} \mathrm{E}$, $29^{\circ} 58^{\prime} \mathrm{N}$ ) in 2017 and 2018 (2017YA and 2018YA) in a randomized block design. Each line was single-seed planted in one row of $2 \mathrm{~m}$ in length with $10 \mathrm{~cm}$ between plants within a row and $30 \mathrm{~cm}$ between rows. Nitrogen and superphosphate fertilizers were applied at a rate of 80 and $100 \mathrm{~kg} / \mathrm{ha}$, respectively, at sowing [19]. Field management was performed according to the common practices for wheat production. At least 3 main spikes of different plants in each line were harvested when ripening.

\section{Phenotypic data}

Thirty kernels of each line were scanned by Epson Expression 10,000 XL. KL and KW were evaluated by WinSEEDLE (Regent Instruments Canada Inc) based on the output images. TKW was calculated as 10 folds of the weight of 100 seeds with three replicates. The other agronomic traits, including spikelet number per spike, spike length, plant height, productive tiller number, kernel number per spike and anthesis date, were investigated with five plants of each line at the corresponding stage as described in previous studies $[24,46]$. Details of investigated traits in different environments were listed in Additional file 9: Table S8. SPSS 22 (IBM SPSS, Armonk, NY, USA) was used for analyzing the phenotypic variance. SAS V8.0 (SAS Institute, Cary, North Carolina) was used for calculating the BLUP for all the investigated traits from different environments. The Pearson correlations between various investigated traits based on the BLUP dataset and between different environments were calculated using SPSS 22. The broad-sense heritability $\left(h^{2}\right)$ across different environments was estimated as described by Smith, et al. [54]. Student's $t$-test $(P<0.05)$ performed by SPSS 22 was used to estimate the significant differences between two parents for three kernel traits.

\section{Map construction and QTL mapping}

The previously constructed genetic map of $2 \mathrm{CN}$ population $[46,55]$ consisted of 34 linkage groups spanning $3005.04 \mathrm{cM}$ and covered all 21 chromosomes of wheat. Here, we integrated the 34 linkage groups into 21 groups covering each of the 21 chromosomes of wheat. The reconstructed genetic map contained 2513 bin markers. The average interval between two adjacent markers is $1.74 \mathrm{cM}$. The A, B, and D subgenomes were 1483.88, 1513.80 and $1372.33 \mathrm{cM}$, with a density of $1.50,1.53$, and $2.58 \mathrm{cM} /$ marker, respectively (Additional file 10: Table S9).

QTL mapping was performed using IciMapping 4.1 based on inclusive composite interval mapping (ICIM). The presence of a QTL was detected above a 3.0 log-of-odds (LOD) threshold. The QE interaction was calculated using data from all the three environments by IciMapping 4.1 with pre-adjusted parameters: Step $=1 \mathrm{cM}, \quad \mathrm{PIN}=0.001$, and $\mathrm{LOD}=3.0$. QTL explained more than $10 \%$ of phenotypic variance and detected in more than 3 environments were considered to be major QTL. QTL were named according to the rules of International Rules of Genetic Nomenclature (http://wheat.pw.usda.gov/ggpages/wgc/98/Intro.htm). 'KL', 'KW', 'TKW' and 'sicau' represent 'kernel length', 'kernel width', 'thousand-kernel weight' and 'Sichuan Agricultural University,' respectively.

\section{Molecular marker analysis}

For KASP marker development, the whole genomic DNA of the parents for the $2 \mathrm{CN}$ population was collected by using the Hi-DNAsecure Plant Kit (Tiangen Biotech Beijing co., Ltd) and further hybridized on the Wheat660K SNP $(630,517)$ genotyping array by CapitalBio Technology Company (Beijing) as descripted previously [24]. Based on genotyping results, a KASP marker was developed in putative QTL regions following standard KASP guidelines (https://www.lgcgroup.com/LGCGroup/media/PDFs/Products/Genotyping/KASP-genotyping-chemistry-User-guide. pdf). The allele-specific forward primers were designed carrying the standard FAM (5'GAAGGTGACCAAGTTCATGCT 3') and HEX (5' GAAGGTCGGAGTCA ACGGATT 3') tails with the targeted SNP at the $3^{\prime}$ end. A common reverse primer was designed with the total amplicon length was $71 \mathrm{bp}$. The detailed primers are listed in Additional file 11: Table S10. Moreover, KASP-AX94721936 was utilized for genotyping $2 \mathrm{CN}$ population. Ten $\mu \mathrm{L}$ PCR reaction mixtures contained $5 \mu \mathrm{l}$ of $1 \times$ KASP mas-

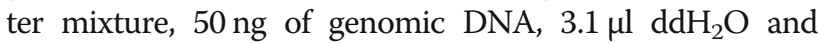
$1.4 \mu \mathrm{l}$ primer mixture (comprised by $30 \mu \mathrm{l}$ reverse primer,

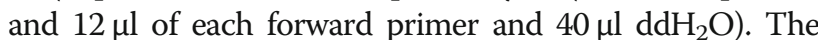
PCR cycling parameters were: hot start at $94{ }^{\circ} \mathrm{C}$ for $15 \mathrm{~min}$, followed by ten touchdown cycles $\left(94^{\circ} \mathrm{C}\right.$ for $20 \mathrm{~s}$; 
touchdown at $61{ }^{\circ} \mathrm{C}$ initially and decreasing by $-0.6^{\circ} \mathrm{C}$ per cycle for $1 \mathrm{~min}$ ), followed by 25 additional cycles of annealing $\left(95^{\circ} \mathrm{C}\right.$ for $20 \mathrm{~s} ; 55^{\circ} \mathrm{C}$ for $\left.1 \mathrm{~min}\right)$. The whole process was carried on real-time PCR (BioRad ${ }^{\circ}$, CFX-96) system. The difference between the homozygous lines of two parental alleles based on the genotyping results was detected using student's $t$-test $(P<0.05)$ with SPSS 22.

\section{Comparison of QTL related to kernel traits}

The genome assembly and coding sequences (CDS) of the wheat cultivar Chinese Spring or 'CS' [IWGSC RefSeq v1.0] [56] were download from https://urgi.versailles.inra. $\mathrm{fr} /$ download/iwgsc/. We used flanking markers of major QTL to BLAST against the pseudomolecules of 'CS' to get their corresponding physical positions. Genes in the target region were retrieved based on CDS (IWGSC_RefSeq_Annotations_v1.0 for 'CS') and were analyzed on UniProt (http://www.uniprot.org/) for annotation and function.

\section{Estimation of effect of 1BL/1RS translocation on kernel traits}

As 'CN16' carries the 1BL/1RS translocation [57], the 1BL/ 1RS translocation of the 2CN RILs derived from '20828' and 'CN16' were previously identified based on the genotype of SNP markers on chromosome 1BS [46]. As nearly no genetic recombination occurred between $1 \mathrm{RS}$ and $1 \mathrm{BS}$, the constructed genetic map did not cover 1BS [55]. We thus estimated the possible effect of 1BL/1RS translocation on kernel traits of $2 \mathrm{CN}$ population. The previous identified lines carrying 1RS (34 lines) and 1BS (139 lines), respectively [46], were compared using student's $t$-test $(P<0.05)$ with SPSS 22.

\section{Supplementary information}

Supplementary information accompanies this paper at https://doi.org/10. 1186/s12863-019-0782-4.

Additional file 1: Figure S1. Effect of 1BL/1RS translocation on kernel traits. '-' represents the homozygous lines carrying 1BS, ' + ' represents the homozygous lines carrying $1 \mathrm{RS}, \mathrm{N}$ represents no significant difference were detected

Additional file 2: Table S1. Correlation coefficients for kernel traits in different environments.

Additional file 3: Table S2. Correlation coefficients among different kernel traits.

Additional file 4: Table S3. Correlation coefficients between agronomic traits and kernel traits in $2 \mathrm{CN}$ population.

Additional file 5: Table S4. Quantitative trait loci detected in the QTL $\times$ environment interaction module.

Additional file 6: Table S5. Comparison of QTL for kernel traits-related genes from different chromosomes identified in this study with those in previous studies.

Additional file 7: Table S6. Predicted genes in the interval of the QTL identified in this study.

Additional file 8: Table S7. QTL mapping for plant height $(\mathrm{PH})$, anthesis date (AD), and spikelet number per spike (SNS) on chromosome 2D.

Additional file 9: Table S8. Details of investigated traits in different environments.
Additional file 10: Table S9. Genetic map of the 2CN mapping population.

Additional file 11: Table S10. Details of KASP markers.

\section{Abbreviations}

'CN16': Chuannong16; 'CS': 'Chinese Spring'; AS-PCR: Allele-specific PCR; BLUP: Best linear unbiased predictors; CAPS: Cleaved amplified polymorphic sequences; CDS: Coding sequences; CZ: Chongzhou; dCAPS: Derived cleaved amplified polymorphic sequences; KASP: Kompetitive allele-specific PCR; KL: Kernel length; KW: Kernel width; QTL: Quantitative trait locus/loci; RIL: Recombinant inbred line; SNP: Single nucleotide polymorphism; TKW: Thousand-kernel weight; YA: Ya'an

\section{Acknowledgments}

Not applicable.

\section{Authors' contributions}

JM initiated the project, designed the experiments, guided the entire study, and extensively revised this manuscript. $\mathrm{HZ}$ performed the research, drafted and revised this manuscript. SQL participated in revising this manuscript and performing the research. YYZ, TL, JJL and PYD performed samples and data collection. YM, HPT and MD performed field work and collected samples. YXL, QTJ, GYC, HYK, ZEP and YMW performed manuscript revision and partial data analysis. YLZ, discussed results and revised this manuscript. $X J L$ guided the entire study, discussed results and revised this manuscript. All authors read and approved the final manuscript.

\section{Funding}

This work is supported by the National Natural Science Foundation of China (31971937 and 31970243), the Key Research and Development Program of Sichuan Province (2018NZDZX0002), and the International Science and Technology Cooperation and Exchanges Program of Science and Technology Department of Sichuan Province (2017HH0076). The funders had no role in study design, data collection and analysis, decision to publish, or the manuscript preparation.

\section{Availability of data and materials}

The datasets supporting the conclusions of this study are included in this published article and its supplementary information files.

\section{Ethics approval and consent to participate}

The research in this study did not involve humans, animals, endangered plants, or engineered plants. All the materials used in this study were provided by Triticeae Research Institute, Sichuan Agricultural University.

\section{Consent for publication}

Not applicable.

\section{Competing interests}

The authors declare that they have no competing interests.

\section{Author details}

${ }^{1}$ Triticeae Research Institute, Sichuan Agricultural University, Chengdu 611130, Sichuan, China. ${ }^{2}$ China State Key Laboratory of Crop Gene Exploration and Utilization in Southwest China, Sichuan Agricultural University, Chengdu 611130, China. ${ }^{3}$ College of Agronomy, Sichuan Agricultural University, Chengdu 611130, Sichuan, China.

Received: 9 September 2019 Accepted: 26 September 2019 Published online: 16 October 2019

\section{References}

1. Ray DK, Mueller ND, West PC, Foley JA. Yield trends are insufficient to double global crop production by 2050. PLoS One. 2013;8(6):e66428.

2. Fischer T, Byerlee D, Edmeades G. Crop yields and food security: will yield increases continue to feed the world. In: Proceedings of the 12th Australian Agronomy Conference; 2012. p. 14-8.

3. Ma L, Li T, Hao C, Wang Y, Chen X, Zhang X. Ta GS 5-3A, a grain size gene selected during wheat improvement for larger kernel and yield. Plant Biotechnol J. 2016;14(5):1269-80. 
4. Su Z, Jin S, Lu Y, Zhang G, Chao S, Bai G. Single nucleotide polymorphism tightly linked to a major QTL on chromosome 7A for both kernel length and kernel weight in wheat. Mol breeding. 2016;36(2):15.

5. Ying J-Z, Ma M, Bai C, Huang $X-H$, Liu J-L, Fan $Y$-Y, Song X-J. TGW3, a major QTL that negatively modulates grain length and weight in rice. Mol Plant. 2018;11(5):750-3.

6. Xia D, Zhou H, Liu R, Dan W, Li P, Wu B, Chen J, Wang L, Gao G, Zhang Q. GL3. 3, a novel QTL encoding a GSK3/SHAGGY-like kinase, epistatically interacts with GS3 to produce extra-long grains in rice. Mol Plant. 2018;11(5):754-6.

7. Hu Z, Lu S-J, Wang M-J, He H, Sun L, Wang H, Liu X-H, Jiang L, Sun J-L, Xin X. A novel QTL qTGW3 encodes the GSK3/SHAGGY-like kinase OsGSK5/ OsSK41 that interacts with OsARF4 to negatively regulate grain size and weight in rice. Mol Plant. 2018;11(5):736-49.

8. Liu Q, Han R, Wu K, Zhang J, Ye Y, Wang S, Chen J, Pan Y, Li Q, Xu X. Gprotein $\beta \gamma$ subunits determine grain size through interaction with MADSdomain transcription factors in rice. Nat Commun. 2018;9(1):852.

9. Weng J, Gu S, Wan X, Gao H, Guo T, Su N, Lei C, Zhang X, Cheng Z, Guo X. Isolation and initial characterization of GW5, a major QTL associated with rice grain width and weight. Cell Res. 2008;18(12):1199.

10. Zhao D-S, Li Q-F, Zhang C-Q, Zhang C, Yang Q-Q, Pan L-X, Ren X-Y, Lu J, Gu $\mathrm{M}-\mathrm{H}$, Liu Q-Q. GS9 acts as a transcriptional activator to regulate rice grain shape and appearance quality. Nat Commun. 2018;9(1):1240.

11. Zhai H, Feng Z, Du X, Song Y, Liu X, Qi Z, Song L, Li J, Li L, Peng H. A novel allele of TaGW2-A1 is located in a finely mapped QTL that increases grain weight but decreases grain number in wheat (Triticum aestivum L.). Theor Appl Genet. 2018;131(3):539-53.

12. Wang S, Yan X, Wang Y, Liu H, Cui D, Chen F. Haplotypes of the TaGS5-A1 gene are associated with thousand-kernel weight in Chinese bread wheat. Front Plant Sci. 2016;7:783.

13. Song X-J, Huang W, Shi M, Zhu M-Z, Lin H-X. A QTL for rice grain width and weight encodes a previously unknown RING-type E3 ubiquitin ligase. Nat Genet. 2007;39(5):623.

14. Yan X, Zhao L, Ren Y, Dong Z, Cui D, Chen F. Genome-wide association study revealed that the TaGW8 gene was associated with kernel size in Chinese bread wheat. SCI rep-Uk. 2019;9(1):2702.

15. Brinton J, Simmonds J, Minter F, Leverington-Waite M, Snape J, Uauy C. Increased pericarp cell length underlies a major quantitative trait locus for grain weight in hexaploid wheat. New Phytol. 2017;215(3):1026-38.

16. Cui F, Fan X, Chen M, Zhang N, Zhao C, Zhang W, Han J, Ji J, Zhao X, Yang $L$. QTL detection for wheat kernel size and quality and the responses of these traits to low nitrogen stress. Theor Appl Genet. 2016;129(3):469-84

17. Li Q, Zhang Y, Liu T, Wang F, Liu K, Chen J, Tian J. Genetic analysis of kernel weight and kernel size in wheat (Triticum aestivum L.) using unconditional and conditional QTL mapping. Mol breeding. 2015;35(10):194.

18. Wu Q-H, Chen Y-X, Zhou S-H, Fu L, Chen J-J, Xiao Y, Zhang D, Ouyang S-H, Zhao X-J, Cui Y. High-density genetic linkage map construction and QTL mapping of grain shape and size in the wheat population Yanda1817X Beinong6. PLoS One. 2015;10(2):e0118144.

19. Yu M, Mao SL, Hou DB, Chen GY, Pu ZE, Li W, Lan XJ, Jiang QT, Liu YX Deng $M$. Analysis of contributors to grain yield in wheat at the individual quantitative trait locus level. Plant Breed. 2018;137(1):35-49.

20. Cui F, Zhao C, Ding A, Li J, Wang L, Li X, Bao Y, Li J, Wang H. Construction of an integrative linkage map and QTL mapping of grain yield-related traits using three related wheat RIL populations. Theor Appl Genet. 2014;127(3):659-75.

21. Uauy C. Wheat genomics comes of age. Curr opini plant biol. 2017;36:142-8.

22. Yang D, Liu Y, Cheng H, Chang L, Chen J, Chai S, Li M. Genetic dissection of flag leaf morphology in wheat (Triticum aestivum L.) under diverse water regimes. BMC Genet. 2016;17(1):94.

23. Hussain W, Baenziger PS, Belamkar V, Guttieri MJ, Venegas JP, Easterly A, Sallam A, Poland J. Genotyping-by-sequencing derived high-density linkage map and its application to QTL mapping of flag leaf traits in bread wheat. SCI rep-UK. 2017:7(1):16394

24. Ma J, Ding P, Liu J, Li T, Zou Y, Habib A, Mu Y, Tang H, Jiang Q, Liu Y. Identification and validation of a major and stably expressed QTL for spikelet number per spike in bread wheat. Theor Appl Genet. 2019. https:/doi.org/10.1007/s00122-019-03415-z.

25. McFadden ES, Sears ER. The origin of Triticum spelta and its free-threshing hexaploid relatives. J Hered. 1946;37(3):81-9.

26. Ogbonnaya FC, Abdalla O, Mujeeb-Kazi A, Kazi AG, Xu SS, Gosman N, Lagudah ES, Bonnett D, Sorrells ME, Tsujimoto H. Synthetic hexaploids: harnessing species of the primary gene pool for wheat improvement. Plant Breed Rev. 2013;37:35-122.
27. Periyannan S, Moore J, Ayliffe M, Bansal U, Wang X, Huang L, Deal K, Luo M, Kong X, Bariana $\mathrm{H}$. The gene Sr33, an ortholog of barley Mla genes, encodes resistance to wheat stem rust race Ug99. Science. 2013;341(6147):786-8.

28. Zhao G, Zou C, Li K, Wang K, Li T, Gao L, Zhang X, Wang H, Yang Z, Liu X. The Aegilops tauschii genome reveals multiple impacts of transposons. Nat Plants. 2017;3(12):946.

29. Huang X, Cloutier S, Lycar L, Radovanovic N, Humphreys D, Noll J, Somers D, Brown P. Molecular detection of QTLs for agronomic and quality traits in a doubled haploid population derived from two Canadian wheats (Triticum aestivum L.). Theor Appl Genet. 2006;113(4):753-66.

30. Ammiraju J, Dholakia B, Santra D, Singh H, Lagu M, Tamhankar S, Dhaliwal H, Rao V, Gupta V, Ranjekar P. Identification of inter simple sequence repeat (ISSR) markers associated with seed size in wheat. Theor Appl Genet. 2001;102(5):726-32.

31. Cuthbert JL, Somers DJ, Brûlé-Babel AL, Brown PD, Crow GH. Molecular mapping of quantitative trait loci for yield and yield components in spring wheat (Triticum aestivum L.). Theor Appl Genet. 2008;117(4):595-608.

32. Huang $X$, Cöster $H$, Ganal M, Röder M. Advanced backcross QTL analysis for the identification of quantitative trait loci alleles from wild relatives of wheat (Triticum aestivum L.). Theor Appl Genet. 2003;106(8):1379-89.

33. Huang $Y$, Kong Z, Wu X, Cheng R, Yu D, Ma Z. Characterization of three wheat grain weight QTLs that differentially affect kernel dimensions. Theor Appl Genet. 2015;128(12):2437-45.

34. Manickavelu A, Kawaura K, Imamura H, Mori M, Ogihara Y. Molecular mapping of quantitative trait loci for domestication traits and $\beta$-glucan content in a wheat recombinant inbred line population. Euphytica. 2011;177(2):179-90.

35. Mohler V, Albrecht T, Castell A, Diethelm M, Schweizer G, Hartl L. Considering causal genes in the genetic dissection of kernel traits in common wheat. J Appl Genet. 2016:57(4):467-76.

36. Wang $R$, Hai L, Zhang X, You G, Yan C, Xiao S. QTL mapping for grain filling rate and yield-related traits in RILs of the Chinese winter wheat population Heshangmaix Yu8679. Theor Appl Genet. 2009;118(2):313-25.

37. Jia H, Li J, Zhu J, Fan T, Qian D, Zhou Y, Wang J, Ren H, Xiang Y, An L. Arabidopsis CROLIN1, a novel plant actin-binding protein, functions in crosslinking and stabilizing actin filaments. J Biol Chem. 2013;288(45):32277-88.

38. Maruyama D, Yamamoto M, Endo T, Nishikawa S-i. Different sets of ERresident J-proteins regulate distinct polar nuclear-membrane fusion events in Arabidopsis thaliana. Plant Cell Physiol. 2014;55(11):1937-44.

39. Ramya P, Chaubal A, Kulkarni K, Gupta L, Kadoo N, Dhaliwal H, Chhuneja P Lagu M, Gupt V. QTL mapping of 1000-kernel weight, kernel length, and kernel width in bread wheat (Triticum aestivum L.). J Appl Genet. 2010;51(4):421-9.

40. Williams K, Sorrells ME. Three-dimensional seed size and shape QTL in hexaploid wheat (Triticum aestivum L.) populations. Crop Sci. 2014;54(1):98-110.

41. Van den Burg HA, Tsitsigiannis DI, Rowland O, Lo J, Rallapalli G, MacLean D, Takken FL, Jones JD. The F-box protein ACRE189/ACIF1 regulates cell death and defense responses activated during pathogen recognition in tobacco and tomato. Plant Cell. 2008;20(3):697-719.

42. Li S, Jia J, Wei $X$, Zhang $X$, Li L, Chen H, Fan Y, Sun H, Zhao X, Lei T. A intervarietal genetic map and QTL analysis for yield traits in wheat. Mol breeding. 2007;20(2):167-78.

43. Pan Z, Ren X, Zhao H, Liu L, Tan Z, Qiu F. A mitochondrial transcription termination factor, ZmSmk3, is required for nad1 intron4 and nad4 intron 1 splicing and kernel development in maize. G3: Genes, Genomes, Genetics. 2019. https://doi.org/10.1534/g3.119.400265.

44. Zhang G, Wang Y, Guo Y, Zhao Y, Kong F, Li S. Characterization and mapping of QTLs on chromosome 2D for grain size and yield traits using a mutant line induced by EMS in wheat. Crop J. 2015;3(2):135-44.

45. Breseghello F, Sorrells ME. QTL analysis of kernel size and shape in two hexaploid wheat mapping populations. Field Crop Res. 2007;101(2):172-9.

46. Liu J, Luo W, Qin N, Ding P, Zhang H, Yang C, Mu Y, Tang H, Liu Y, Li W. A 55 K SNP array-based genetic map and its utilization in QTL mapping for productive tiller number in common wheat. Theor Appl Genet. 2018;131(11):2439-50.

47. Griffiths S, Wingen L, Pietragalla J, Garcia G, Hasan A, Miralles D, Calderini DF, Ankleshwaria JB, Waite ML, Simmonds J. Genetic dissection of grain size and grain number trade-offs in CIMMYT wheat germplasm. PLoS One. 2015; 10(3):e0118847.

48. Pinthus MJ, Millet E. Interactions among number of spikelets, number of grains and grain weight in the spikes of wheat (Triticum aestivum L.). Ann Bot. 1978:42(4):839-48.

49. Calderini D, Savin R, Abeledo L, Reynolds M, Slafer G. The importance of the period immediately preceding anthesis for grain weight determination in wheat. Euphytica. 2001;119(1-2):199-204. 
50. Korzun V, Röder M, Ganal M, Worland A, Law C. Genetic analysis of the dwarfing gene (Rht8) in wheat. Part I. molecular mapping of Rht8 on the short arm of chromosome 2D of bread wheat (Triticum aestivum L.). Theor Appl Genet. 1998;96(8):1104-9.

51. Rasheed A, Xia X, Mahmood T, Quraishi UM, Aziz A, Bux H, Mahmood Z, Mirza J, Mujeeb-Kazi A, He Z. Comparison of economically important loci in landraces and improved wheat cultivars from Pakistan. Crop Sci. 2016;56(1):287-301.

52. Bentley AR, Scutari M, Gosman N, Faure S, Bedford F, Howell P, Cockram J, Rose GA, Barber T, Irigoyen J. Applying association mapping and genomic selection to the dissection of key traits in elite European wheat. Theor Appl Genet. 2014;127(12):2619-33.

53. Semagn K, Babu R, Hearne S, Olsen M. Single nucleotide polymorphism genotyping using Kompetitive allele specific PCR (KASP): overview of the technology and its application in crop improvement. Mol breeding. 2014;33(1):1-14.

54. Smith SE, Kuehl R, Ray I, Hui R, Soleri D. Evaluation of simple methods for estimating broad-sense heritability in stands of randomly planted genotypes. Crop Sci. 1998:38(5):1125-9.

55. Ma J, Qin N, Cai B, Chen G, Ding P, Zhang H, Yang C, Huang L, Mu Y, Tang H. Identification and validation of a novel major QTL for all-stage stripe rust resistance on 1BL in the winter wheat line 20828. Theor Appl Genet. 2019; 132(5):1363-73.

56. Appels R, Eversole K, Feuillet C, Keller B, Rogers J, Stein N, Pozniak CJ, Choulet F, Distelfeld A, Poland J. Shifting the limits in wheat research and breeding using a fully annotated reference genome. Science. 2018; 361 (6403):eaar7191.

57. Qi W, Tang Y, Zhu W, Li D, Diao C, Xu L, Zeng J, Wang Y, Fan X, Sha L. Molecular cytogenetic characterization of a new wheat-rye 1BL 1 RS translocation line expressing superior stripe rust resistance and enhanced grain yield. Planta. 2016;244(2):405-16.

\section{Publisher's Note}

Springer Nature remains neutral with regard to jurisdictional claims in published maps and institutional affiliations.

Ready to submit your research? Choose BMC and benefit from:

- fast, convenient online submission

- thorough peer review by experienced researchers in your field

- rapid publication on acceptance

- support for research data, including large and complex data types

- gold Open Access which fosters wider collaboration and increased citations

- maximum visibility for your research: over $100 \mathrm{M}$ website views per year

At $\mathrm{BMC}$, research is always in progress.

Learn more biomedcentral.com/submissions 